Article

\title{
Expression Profile of the Chromosome 14 MicroRNA Cluster (C14MC) Ortholog in Equine Maternal Circulation throughout Pregnancy and Its Potential Implications
}

\author{
Pouya Dini ${ }^{1,2,+} \oplus$, Hossam El-Sheikh Ali ${ }^{2,3,+} \oplus$, Mariano Carossino ${ }^{2,4}$, Shavahn C. Loux ${ }^{2}$, \\ A. Esteller-Vico ${ }^{5}$, Kirsten E. Scoggin ${ }^{2}$, Peter Daels ${ }^{1}\left(\mathbb{D}\right.$ and Barry A. Ball ${ }^{2, *}$ (D) \\ 1 Faculty of Veterinary Medicine, Ghent University, 9820 Merelbeke, Belgium; pouya.dini@uky.edu (P.D.); \\ Peter.Daels@UGent.be (P.D.) \\ 2 Gluck Equine Research Center, Department of Veterinary Science, University of Kentucky, \\ Lexington, KY 40503, USA; hossam.elsheikh@uky.edu (H.E.-S.A.); mcarossino1@lsu.edu (M.C.); \\ shavahn.loux@uky.edu (S.C.L.); kirsten.scoggin@uky.edu (K.E.S.) \\ 3 Theriogenology Department, Faculty of Veterinary Medicine, University of Mansoura, \\ Mansoura 35516, Egypt \\ 4 Louisiana Animal Disease Diagnostic Laboratory and Department of Pathobiological Sciences, School of \\ Veterinary Medicine, Louisiana State University, Baton Rouge, LA 70803, USA \\ 5 Department of Biomedical and Diagnostic Sciences, College of Veterinary Medicine, University of Tennessee, \\ Knoxville, TN 37996, USA; aestellervico@uky.edu \\ * Correspondence: b.a.ball@uky.edu; Tel.: +01x-859-218-1141 \\ + These authors contributed equally to this work.
}

Received: 8 November 2019; Accepted: 7 December 2019; Published: 13 December 2019

Abstract: Equine chromosome 24 microRNA cluster (C24MC), the ortholog of human C14MC, is a pregnancy-related miRNA cluster. This cluster is believed to be implicated in embryonic, fetal, and placental development. The current study aimed to characterize the expression profile of this cluster in maternal circulation throughout equine gestation. The expression profile of miRNAs belonging to this cluster was analyzed in the serum of non-pregnant (diestrus), pregnant ( $25 \mathrm{~d}$, $45 \mathrm{~d}, 4 \mathrm{mo}, 6 \mathrm{mo}, 10 \mathrm{mo}$ ), and postpartum mares. Among the miRNAs examined, $11 \mathrm{miRNAs}$ were differentially expressed across the analyzed time-points. Four of these miRNAs (eca-miR-1247-3p, eca-miR-134-5p, eca-miR-382-5p, and eca-miR-433-3p) were found to be enriched in the serum of pregnant mares at Day 25 relative to non-pregnant mares. To further assess the accuracy of these miRNAs in differentiating pregnant $(25 \mathrm{~d})$ from non-pregnant mares, receiver operating characteristic (ROC) analysis was performed for each of these miRNAs, revealing that eca-miR-1247-3p and eca-miR-134-5p had the highest accuracy $\left(\mathrm{AUC}_{\mathrm{ROC}}=0.92\right.$ and 0.91 , respectively; $\left.p<0.05\right)$. Moreover, eca-miR-1247-3p, eca-miR-134-5p, eca-miR-409-3p, and eca-miR-379-5p were enriched in the serum of Day 45 pregnant mares. Among those miRNAs, eca-miR-1247-3p and eca-miR-409-3p retained the highest accuracy as shown by ROC analysis. GO analysis revealed that these miRNAs are mainly implicated in nervous system development as well as organ development. Using in situ hybridization, we localized eca-miR-409-3p in the developing embryo ( $25 \mathrm{~d}$ ) and extra-embryonic membranes ( 25 and $45 \mathrm{~d}$ ). In conclusion, the present study is the first to elucidate the circulating maternal profile of C24MC-associated miRNAs throughout pregnancy and to suggest that serum eca-miR-1247-3p, $e c a-m i R-134-5 p$, and eca-miR-409-3p could be used as pregnancy-specific markers during early gestation ( 25 and $45 \mathrm{~d}$ ). Overall, the high abundance of these embryo-derived miRNAs in the maternal circulation suggests an embryo-maternal communication during the equine early pregnancy. 
Keywords: C14MC; C24MC; equine chromosome 24; pregnancy; circulating microRNAs; biomarker; mare; embryo

\section{Introduction}

MicroRNAs (miRNAs) are a class of small non-coding RNA that post-transcriptionally regulate protein-coding mRNAs [1]. MiRNAs are expressed in a variety of cell types as biological regulators. They play a fundamental role in the regulation of a variety of developmental and physiological processes, such as cell proliferation, growth, metabolism, communication, apoptosis, and death [2-4]. Aberrant expression of miRNAs has been associated with many pathological disorders and diseases, including cancer and abnormalities during pregnancy [5-8]. Occasionally throughout the genome, a group of two or more miRNAs are transcribed from physically adjacent miRNA genes (within $10 \mathrm{~kb}$ ), which form a cluster $[9,10]$. These miRNA clusters have been found to be exclusively or preferentially expressed in a tissue-specific manner [11]. This tissue expression is at least partly reflected in the circulation (i.e., circulating miRNAs) and could be detected in plasma and serum with much greater sensitivity than protein markers [12,13]. The measurability, sensitivity, and stability (approximately five day half-life) of circulating miRNAs $[13,14]$ empowered them to emerge as biomarkers for several physiological and pathological conditions, including diabetes [15], cancer [16,17], cardiovascular diseases [18,19], and skeletal diseases [20].

In reproductive biology, miRNAs are involved in folliculogenesis, corpus luteum formation, endometrial functions, embryogenesis, maternal recognition of pregnancy, embryo implantation, as well as placental development [21-29]. Recently, there has been growing interest in pregnancy-related and/or placenta-specific miRNAs in mammals [7,21,30-33]. This interest led to the identification of several circulating miRNAs as biomarkers for early pregnancy diagnosis [34], prediction and/or diagnosis of embryonic loss [35,36], ectopic pregnancy [37,38], pre-eclampsia [39,40], intrauterine growth restriction [40,41], placental infection [41], and preterm labor [41,42]. In humans, the chromosome 14 miRNA cluster (C14MC) is one of the largest pregnancy-related miRNA clusters and consists of 52 miRNAs [21]. In the horse, C24MC is the orthologous cluster to human C14MC [29]. Recently, our group has elucidated the kinetics of C24MC in the chorioallantoic membrane (CAM) throughout equine gestation [29]. We demonstrated that C24MC-associated miRNAs were upregulated in CAM during the early stages of equine pregnancy, followed by a downregulation later in gestation [29]. Moreover, functional analysis of mRNAs targeted by this miRNAs cluster suggested that C24MC are involved in embryonic development, endothelial cell migration, and angiogenesis during placental development in the horse [29]. So far, the expression profile of C14MC in maternal circulation throughout gestation has not been elucidated.

We hypothesized that equine C24MC-associated miRNAs, the ortholog of human C14MC, will have a differential expression pattern in maternal circulation throughout gestation. Moreover, identifying the normal profile of these miRNAs in maternal circulation in comparison to non-pregnant mares and postpartum mares could positively impact the development of novel and non-invasive biomarkers for pregnancy diagnosis and prediction of pregnancy outcomes and/or complications. Therefore, this study was designed to evaluate the expression profile of equine C24MC-associated miRNAs in the serum of non-pregnant (diestrus), pregnant ( 25 days, 45 days, 4 months, 6 months, 10 months) and postpartum mares.

\section{Results}

\subsection{Equine C24MC Expression in Serum}

To determine the expression pattern of C24MC-associated miRNAs in maternal serum, the expression of candidate miRNAs was evaluated in samples from pregnant ( $25 \mathrm{~d}, 45 \mathrm{~d}, 4 \mathrm{mo}$, 
$6 \mathrm{mo}$, and $10 \mathrm{mo}$ ), postpartum and non-pregnant (diestrous) mares. Out of the 26 tested miRNAs, 13 of them showed a persistent expression at all time-points (expressed in all the samples from at least one time points). The remaining 13 miRNAs were not detected in all the samples therefore were excluded from the study. Regarding the abundance of tested miRNAs in equine circulation, we found eca-miR-433-3p, eca-miR-1247-3p, eca-miR-134-5p, and eca-miR-411-5p with the greatest abundance in serum $(p<0.05)$. On the other hand, eca-miR-412-5p and eca-miR-379-5p showed the lowest abundance $(p<0.05)$ (Figure 1). Out of the tested miRNAs, 11 miRNAs were differentially expressed across the analyzed time-points $(p<0.05)$, whereas the expression profile of eca-miR-412-5p and eca-miR-432-5p did not show any significant fluctuation. The differential expression profile of tested miRNAs is depicted in Figures 2 and 3. The correlation between tested miRNAs expression profiles is illustrated in Table 1.

Among the differentially expressed miRNAs, four miRNAs (eca-miR-1247-3p, eca-miR-134-5p, eca-miR-382-5p, and eca-miR-433-3p) were found to be enriched $(p<0.05)$ in the serum of Day 25 pregnant mares in comparison to non-pregnant (diestrous) mares, as illustrated in Figure 2. Another common feature among these miRNAs is the downregulation $(p<0.05)$ of all of them at 10 mo of gestation in comparison to $25 \mathrm{~d}$ of gestation (Figure 2). Additionally, the expression profile of all the expressed miRNAs was positively correlated to each other, with the highest correlation observed between eca-miR-1247-3p and eca-miR-433-3p ( $\mathrm{r}=0.92, p<0.01$; Table 1$)$. To determine the accuracy of these miRNAs in distinguishing pregnant $(25 \mathrm{~d})$ from non-pregnant mares, ROC analysis was performed and demonstrated that eca-miR-1247-3p and eca-miR-134-5p showed the highest accuracy (AUC ROC $_{1}$ $=0.92$ and 0.91 , respectively; $p<0.01$ ) compared to eca-miR-382-5p and eca-miR-433-3p, which were moderately accurate $\left(\mathrm{AUC}_{\mathrm{ROC}}=0.88\right.$ and 0.83 , respectively; $p<0.05$ ) (Figure $\left.4 \mathrm{~A}\right)$. Out of these four miRNAs, eca-miR-1247-3p and eca-miR-134-5p remained enriched $(p<0.05)$ in serum of Day 45 pregnant mares in comparison to non-pregnant mares. Similarly, eca-miR-409-3p and eca-miR-379-5p expression profiles were enriched in serum of Day 45 pregnant mares in comparison to non-pregnant mares $(p<0.05)$. Again, ROC analysis was performed to determine the accuracy of these miRNAs in differentiating pregnant $(45 \mathrm{~d})$ from non-pregnant mares, and indicated that eca-miR-1247-3p and eca-miR-409-3p have a significant moderate accuracy $\left(\mathrm{AUC}_{\mathrm{ROC}}=0.81\right.$ and 0.77 , respectively; $p<0.05$ ) relative to a non-significant $\mathrm{AUC}_{\mathrm{ROC}}$ for eca-miR-134-5p $(p=0.05)$ and eca-miR-379-5p $(p=0.284)$, as shown in Figure 4B.

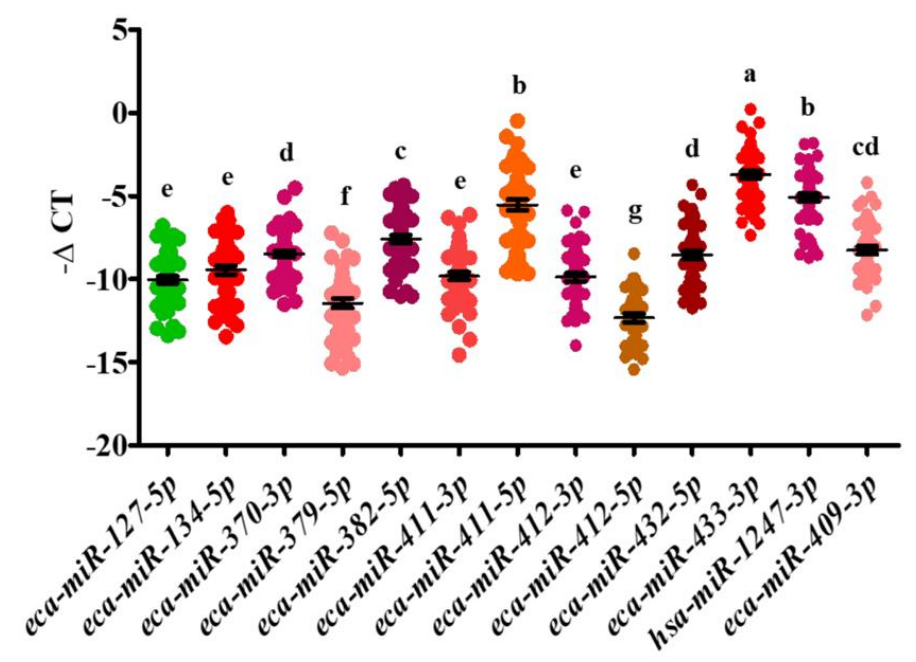

Figure 1. Abundance of the tested miRNAs in equine circulation. Expression of circulating miRNAs was analyzed using RT-qPCR through gestation ( $25 \mathrm{~d}, 45 \mathrm{~d}, 4 \mathrm{mo}, 6 \mathrm{mo}$, and $10 \mathrm{mo}$ ), diestrus (non-pregnant) and post-partum period. Expression of each microRNA was normalized to the geometric mean of eca-miR-10a, eca-miR-21, and eca-Let-7a, expressed as $-\Delta \mathrm{CT}$. Data are presented as a dot plot and the middle horizontal line represents the mean while error bars represent the standard error of the mean (SEM). Significantly, different samples are indicated by varying superscripts. 
A)

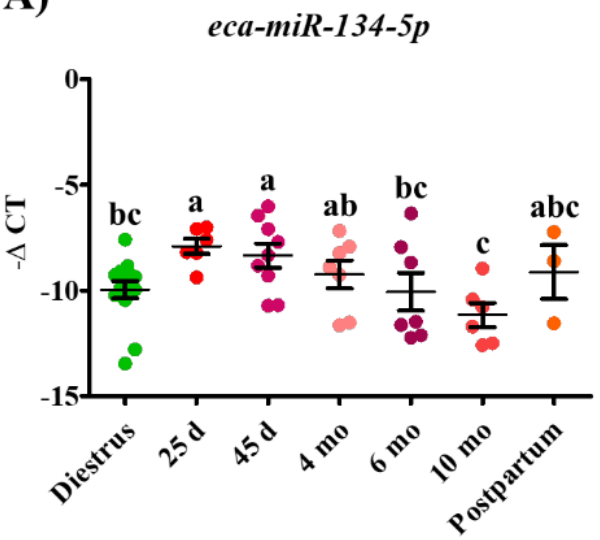

C)

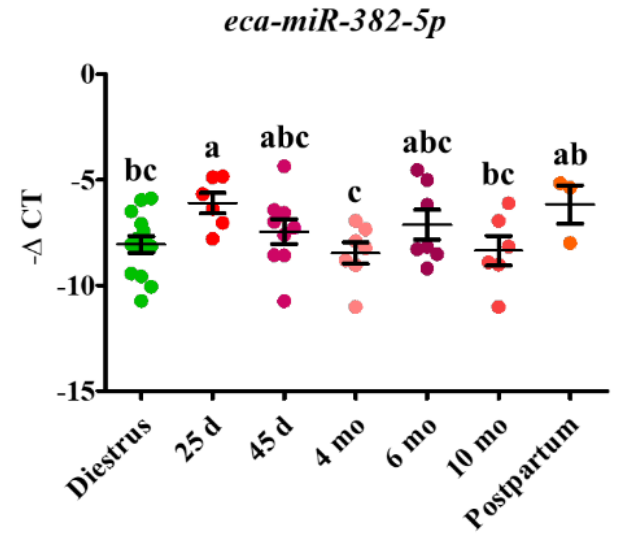

E)

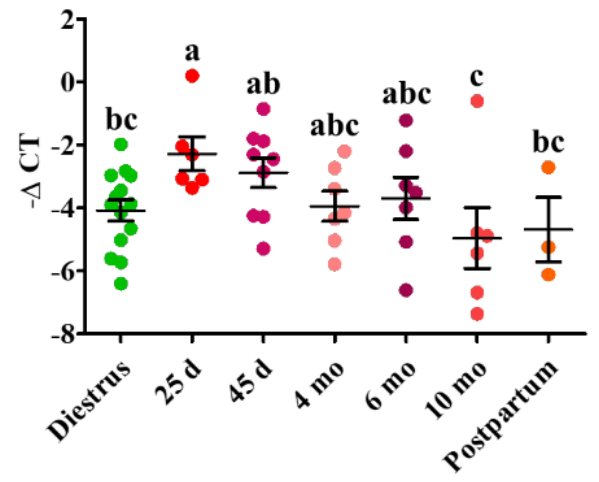

B)

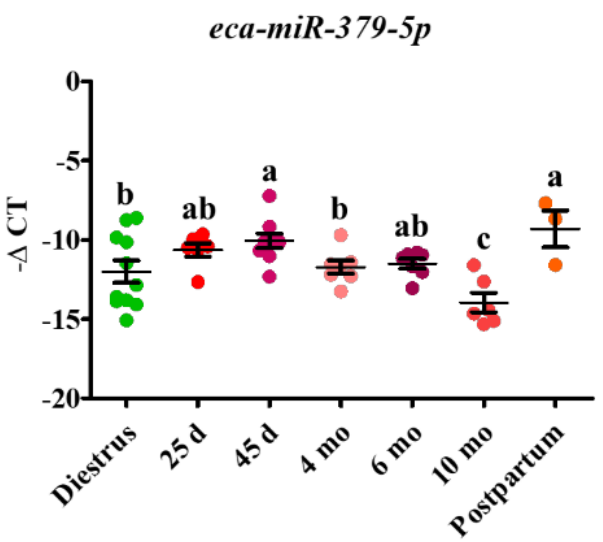

D)

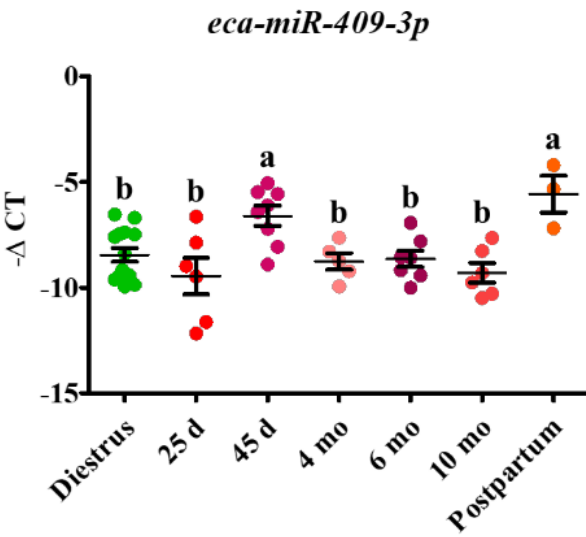

F)

$$
\text { eca-miR-1247-3p }
$$

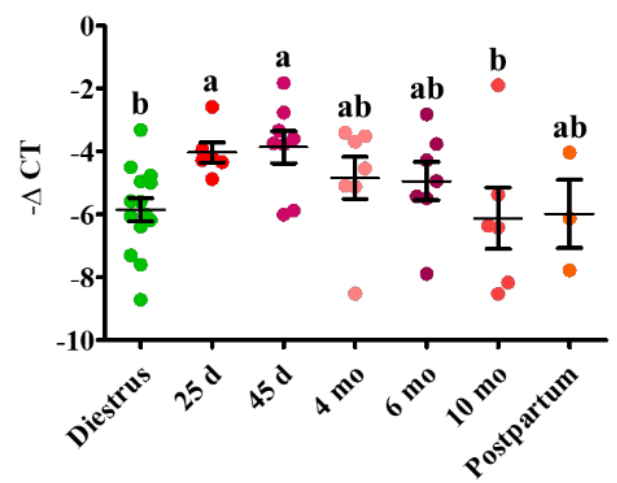

Figure 2. Differential expression profile of eca-miR-134-5p (A), eca-miR-379-5p (B), eca-miR-382-5p (C), eca-mir-409-3p (D), eca-miR-433-3p (E) and eca-miR-1247-3p (F) during diestrus, gestation ( $25 \mathrm{~d}, 45 \mathrm{~d}$, $4 \mathrm{mo}, 6 \mathrm{mo}$ and $10 \mathrm{mo}$ ) and postpartum period in mares' circulation. Expression of each microRNA was normalized to the geometric mean of eca-miR-10a, eca-miR-21, and eca-Let-7a, expressed as $-\triangle \mathrm{CT}$. Data are presented as a dot plot and the middle horizontal line represents the mean while error bars represent the standard error of the mean (SEM). Significantly different time-points are indicated by varying superscripts. 
A)

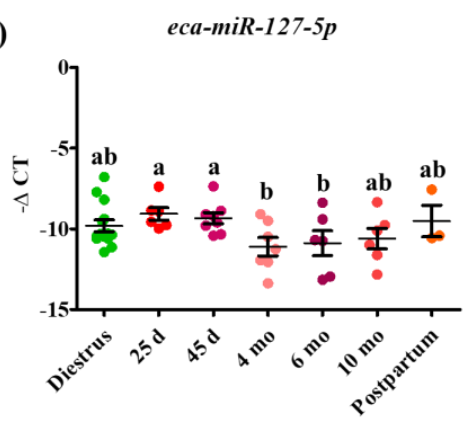

C)

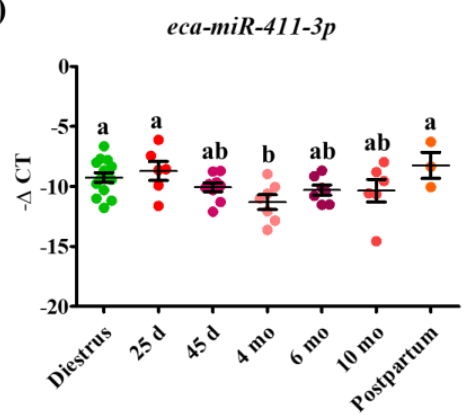

E)

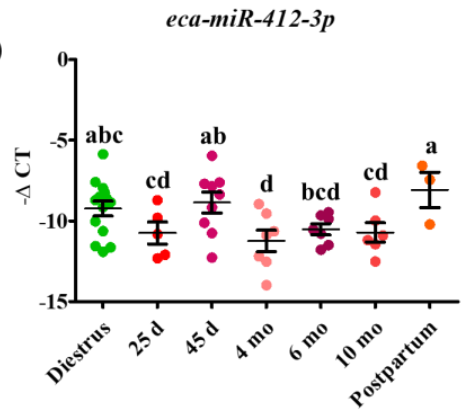

G)

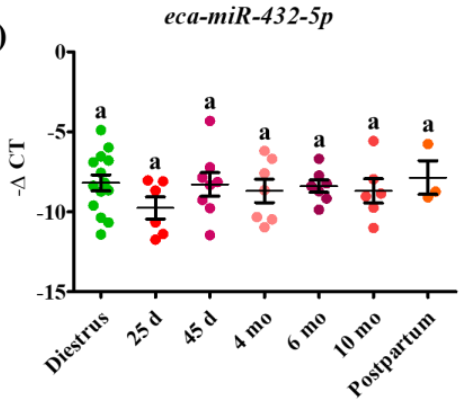

B) eca-miR-370-3p

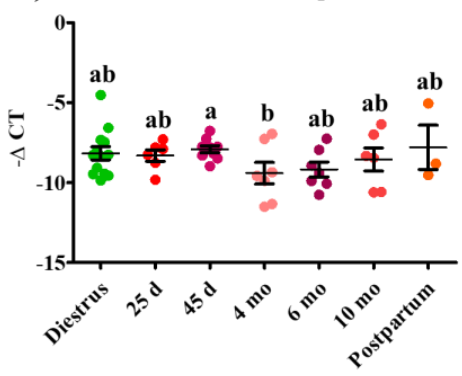

D)
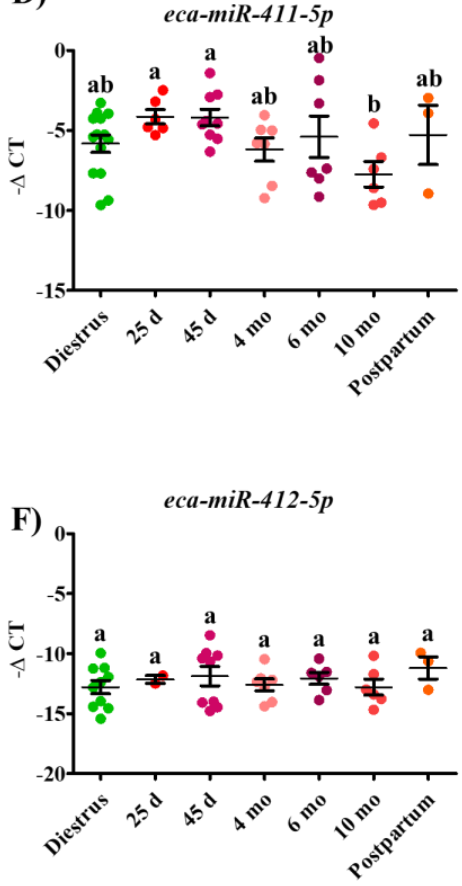

Figure 3. Differential expression profile of eca-miR-127-3p (A), eca-miR-370-3p (B), eca-miR-411-3p (C), eca-miR-411-5p (D), eca-miR-412-3p (E), eca-miR-412-5p (F) and eca-miR-432-5p (G) during diestrus, gestation ( $25 \mathrm{~d}, 45 \mathrm{~d}, 4 \mathrm{mo}, 6 \mathrm{mo}$, and $10 \mathrm{mo}$ ) and postpartum period in mares circulation. Expression of each microRNA was analyzed using RT-qPCR and normalized to the geometric mean of eca-miR-10a, eca-miR-21, and eca-Let-7a, expressed as $-\Delta \mathrm{CT}$. Data are presented as a dot plot and the middle horizontal line represents the mean while error bars represent the standard error of the mean (SEM). Significantly different time-points are indicated by varying superscripts. 
Table 1. The correlation between tested miRNAs expression profiles.

\begin{tabular}{|c|c|c|c|c|c|c|c|c|c|c|c|c|}
\hline & $\begin{array}{c}e c a-m i R- \\
134-5 p\end{array}$ & $\begin{array}{c}e c a-m i R- \\
370-3 p\end{array}$ & $\begin{array}{c}e c a-m i R- \\
379-5 p\end{array}$ & $\begin{array}{c}e c a-m i R- \\
382-5 p\end{array}$ & $\begin{array}{c}e c a-m i R- \\
411-3 p\end{array}$ & $\begin{array}{c}e c a-m i R- \\
411-5 p\end{array}$ & $\begin{array}{c}e c a-m i R- \\
412-3 p\end{array}$ & $\begin{array}{c}e c a-m i R- \\
412-5 p\end{array}$ & $\begin{array}{c}e c a-m i R- \\
432-5 p\end{array}$ & $\begin{array}{c}e c a-m i R- \\
433-3 p\end{array}$ & $\begin{array}{c}h s a-m i R- \\
1247-3 p\end{array}$ & $\begin{array}{c}e c a-m i R \\
409-3 p\end{array}$ \\
\hline eca-miR-127-5p & $0.448^{* *}$ & $0.713^{* *}$ & 0.307 * & $0.348^{*}$ & $0.527 * *$ & $0.428 * *$ & $0.493^{* *}$ & 0.101 & 0.163 & $0.405^{* *}$ & 0.310 * & 0.101 \\
\hline eca-miR-134-5p & 1 & 0.236 & $0.482^{* *}$ & $0.520 * *$ & 0.255 & 0.882 ** & $0.326^{*}$ & 0.079 & 0.23 & $0.597^{* *}$ & $0.614^{* *}$ & 0.313 * \\
\hline eca-miR-370-3p & & 1 & 0.236 & 0.261 & $0.499 * *$ & 0.241 & $0.512^{* *}$ & 0.207 & 0.282 * & 0.296 * & 0.208 & 0.21 \\
\hline eca-miR-379-5p & & & 1 & $0.560^{* *}$ & 0.259 & $0.562 * *$ & $0.511^{* *}$ & 0.251 & 0.242 & 0.253 & 0.303 * & $0.510^{* *}$ \\
\hline eca-miR-382-5p & & & & 1 & $0.498^{* *}$ & $0.641^{* *}$ & $0.393^{* *}$ & 0.053 & $0.295^{*}$ & $0.385^{* *}$ & $0.357^{* *}$ & 0.211 \\
\hline$e c a-m i R-411-3 p$ & & & & & 1 & $0.314^{*}$ & $0.457^{* *}$ & 0.074 & 0.122 & 0.289 * & 0.162 & 0.157 \\
\hline eca-miR-411-5p & & & & & & 1 & $0.362 * *$ & 0.058 & $0.326^{*}$ & $0.634^{* *}$ & $0.636^{* *}$ & 0.246 \\
\hline eca-miR-412-3p & & & & & & & 1 & 0.287 & 0.187 & 0.073 & 0.063 & $0.468^{* *}$ \\
\hline eca-miR-412-5p & & & & & & & & 1 & -0.012 & 0.208 & 0.239 & 0.135 \\
\hline eca-miR-432-5p & & & & & & & & & 1 & 0.236 & $0.316^{*}$ & $0.461^{* *}$ \\
\hline eca-miR-433-3p & & & & & & & & & & 1 & 0.919 ** & 0.053 \\
\hline esa-miR-1247-3p & & & & & & & & & & & 1 & 0.108 \\
\hline
\end{tabular}

${ }^{*} p<0.05 ;{ }^{* *} p<0.01$. 


\section{A)}

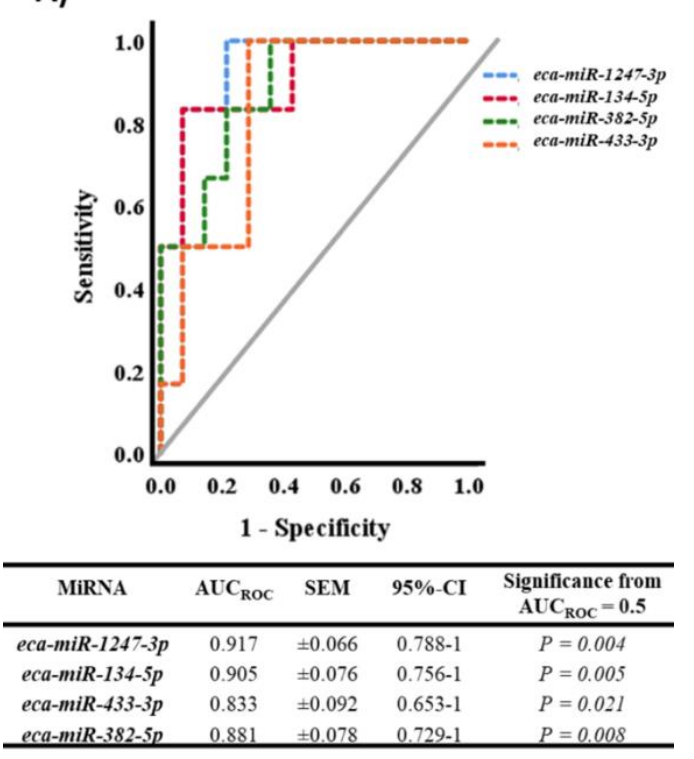

B)

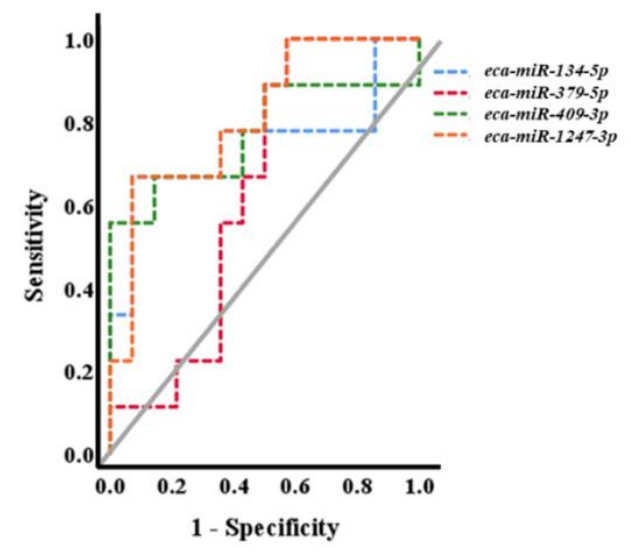

\begin{tabular}{ccccc}
\hline MiRNA & AUC $_{\text {ROC }}$ & SEM & $\mathbf{9 5 \% - C I}$ & $\begin{array}{c}\text { Significance from } \\
\text { AUC }_{\text {ROC }}=\mathbf{0 . 5}\end{array}$ \\
\hline eca-miR-1247-3p & 0.810 & \pm 0.094 & $0.626-0.993$ & $P=0.014$ \\
eca-miR-409-3p & 0.770 & \pm 0.117 & $0.541-0.999$ & $P=0.032$ \\
eca-miR-134-5p & 0.746 & \pm 0.122 & $0.507-0.985$ & $P=0.051$ \\
eca-miR-379-5p & 0.635 & \pm 0.118 & $0.405-0.865$ & $P=0.284$ \\
\hline
\end{tabular}

Figure 4. (A) Receiver operating characteristic (ROC) curves of circulating eca-miR-1247-3p, eca-miR-134-5p, eca-miR-433-3p, and eca-miR-382-5p expression for the discrimination of early pregnant mares $(25 \mathrm{~d}, \mathrm{n}=6$ ) from non-pregnant mares (diestrus, $\mathrm{n}=14)$. (B) ROC curves of circulating eca-miR-1247-3p, eca-miR-134-5p, eca-mir-409-3p, and eca-miR-379-5p expression for the discrimination of early pregnant mares ( $45 \mathrm{~d}, \mathrm{n}=8$ for eca-miR-1247-3p and eca-mir-409-3p, and $\mathrm{n}=9$ for eca-miR-134-5p and eca-miR-379-5p) from non-pregnant mares (diestrus, $\mathrm{n}=14$ ). Table in subfigure $\mathbf{A}$ and $\mathbf{B}$ elucidate the results generated from ROC curve analysis for 25 and $45 \mathrm{~d}$ pregnancy dataset, respectively.

Within the remaining differentially expressed miRNAs, expression of eca-miR-127-5p and eca-miR-411-3p was significantly higher in serum at $25 \mathrm{~d}$ compared to $4 \mathrm{mo}$, as illustrated in Figure 3 . Moreover, eca-miR-127-5p, eca-miR-370-3p , and eca-miR-412-3p were significantly upregulated in serum at $45 \mathrm{~d}$ GA compared to 4 mo GA (Figure 3). 


\subsection{Gene Ontology Analysis for the Differnetially Expressed miRNAs}

Computational target prediction for miRNAs was performed to identify putative mRNA targets. A total of 428 target mRNAs were predicted using mirDB.org and IPA for the differentially expressed miRNAs (eca-miR-134-5p, eca-miR-1247-3p, eca-miR-382-5p, eca-miR-409-3p, eca-miR-433-3p, and eca-miR-379-5p). GO enrichment (biological process) analysis and pathway analysis were carried out. GO analysis revealed that these miRNAs are mainly implicated in nervous system development (Figure 5A). Pathway analysis of the same set of miRNAs revealed that they are associated with the Wnt signaling pathway and oxytocin receptor mediated signaling pathway, among others (Figure 5B).

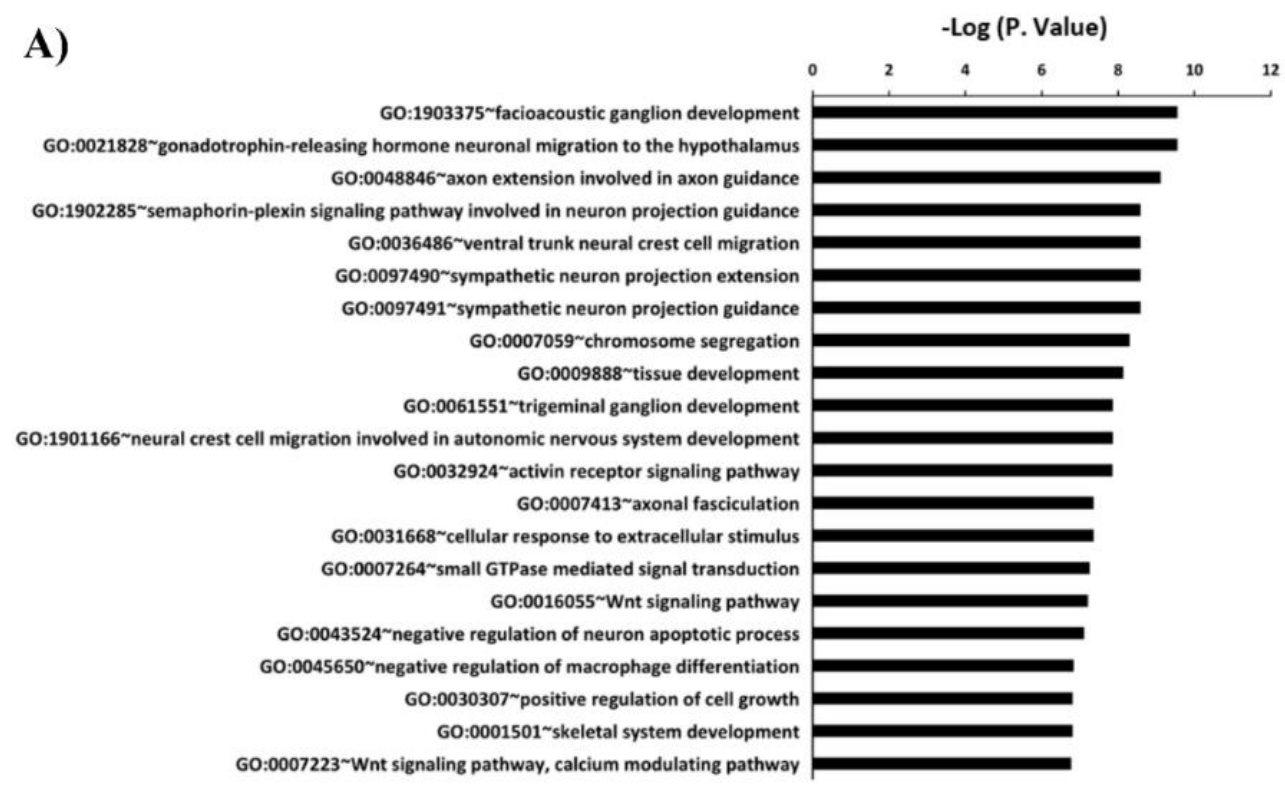

B)

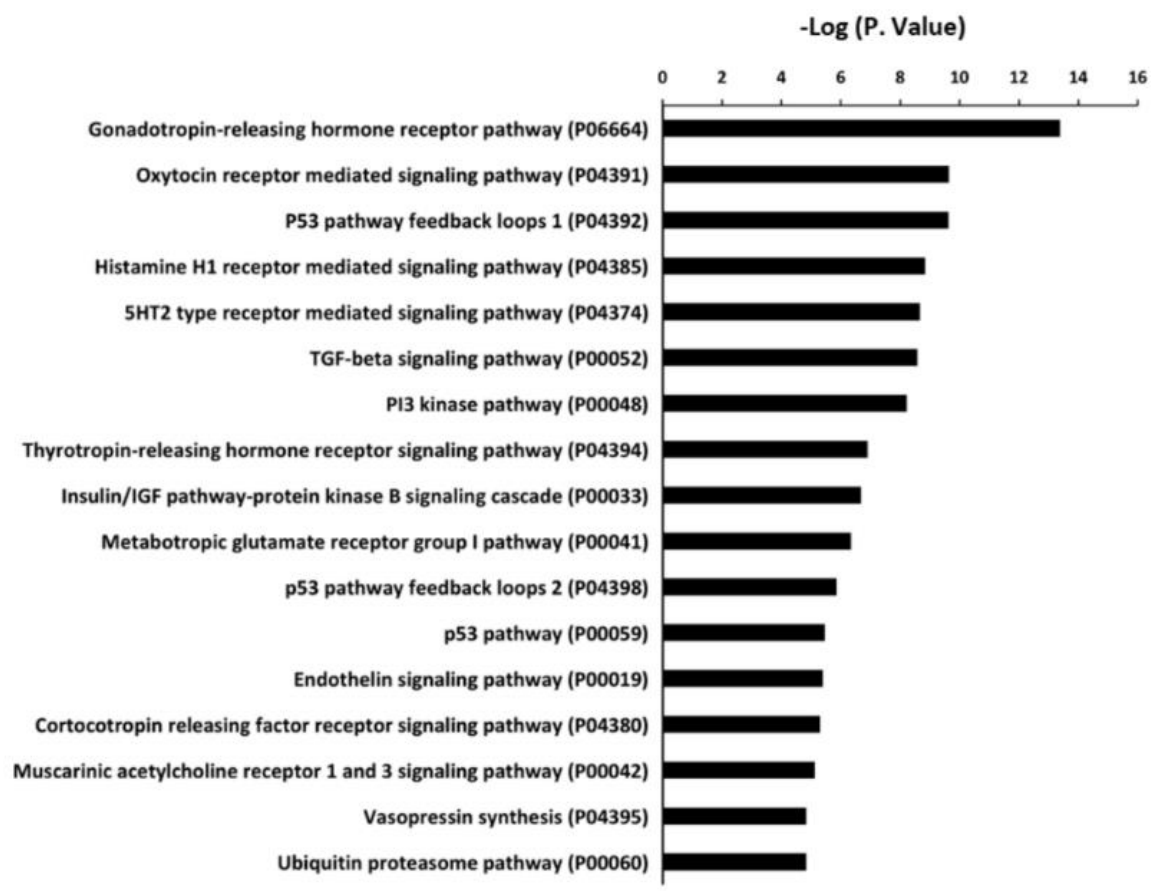

Figure 5. Gene ontology and pathway analysis of C24MC-associated miRNAs. The target mRNAs for eca-miR-1247-3p, eca-miR-134-5p, eca-miR-382-5p, eca-miR-433-3p, eca-miR-409-3p, and eca-miR-379-5p were predicted using IPA and miRDB. (A) Functional annotation analysis (Biological process) of the 428 predicted mRNA' targets using DAVID. (B) Pathways predicted for mRNA's targets using PANTHER. 


\subsection{Localization of a Member of Equine C24MC in Equine Extra-Embryonic Membranes and $25 d$ Embryos}

In order to study the cellular and subcellular localization of eca-miR-409-3p (a member of the C24MC), we performed miRNA in situ hybridization (ISH) on $25 \mathrm{~d}$ embryos along with the extra-embryonic membranes of the 25 and $45 \mathrm{~d}$ GA. Since eca-miR-409-3p had a differential expression pattern during pregnancy and presented a $100 \%$ homology to $h s a-m i R-409-3 p$, this miRNA was chosen as the best candidate for ISH analysis. At $25 \mathrm{~d}$, eca-miR-409-3p was intensely expressed in extra-embryonic membranes (Figure 6). In $25 \mathrm{~d}$ embryos, the expression of this miRNA was extensive in several developing tissues from the three germ layers (Figure 6). Moreover, extra-embryonic membranes collected at 25 and $45 \mathrm{~d}$ shows strong cytoplasmic signal for eca-miR-409-3p specifically in trophoblastic cells (Figure 7).

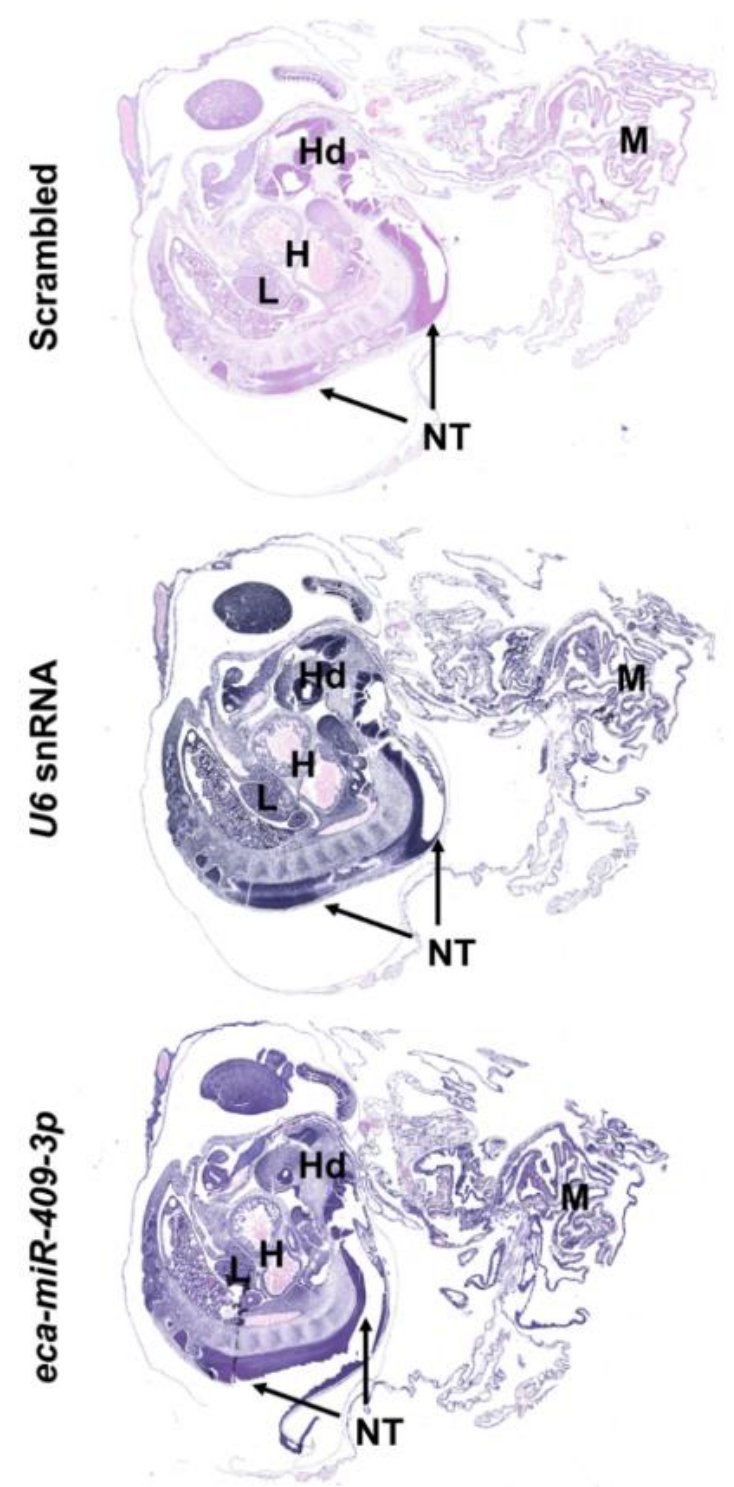

Figure 6. Localization of eca-miR-409-3p in the $25 \mathrm{~d}$ embryo and extra-embryonic membranes ( $25 \mathrm{~d}$ ) by in situ hybridization (bottom image). Extra-embryonic membranes show strong, diffuse signal. Numerous embryonic tissues from the three germ layers including the developing central nervous system (neural tube) show strong signal for eca-miR-409-3p. Scrambled and U6 snRNA were used as negative and positive controls. $M$, extra-embryonic membranes; ${ }^{*}$, head; $H$, heart; NT, neural tube; L, liver. NBT/BCIP (blue) was used as the substrate. Magnification: $25 \times$. 


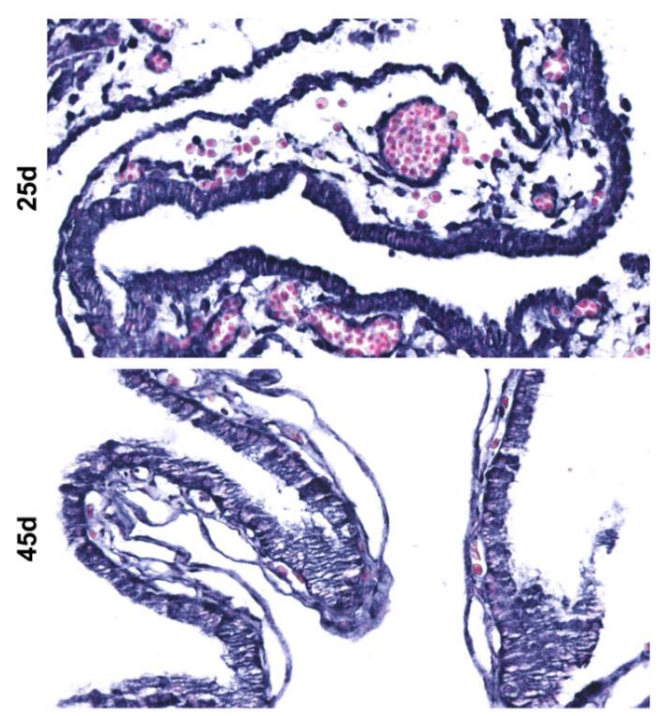

Figure 7. Localization of eca-miR-409-3p in the 25 and $45 \mathrm{~d}$ extra-embryonic membranes by in situ hybridization. Extra-embryonic membranes show strong cytoplasmic signal for eca-miR-409-3p, including trophoblastic cells. NBT/BCIP (blue) was used as the substrate. Magnification: 400×.

\section{Discussion}

To the best of our knowledge, this is the first study elucidating the expression profile of equine C24MC-associated miRNAs in peripheral circulation of non-pregnant (diestrus), pregnant ( $25 \mathrm{~d}$, $45 \mathrm{~d}, 4 \mathrm{mo}, 6 \mathrm{~m}$, and $10 \mathrm{mo}$ ), and postpartum mares. In the present study, four equine miRNAs (eca-miR-1247-3p, eca-miR-134-5p, eca-miR-382-5p, and eca-miR-433-3p) were found to be enriched in the serum of Day 25 pregnant mares in comparison to non-pregnant mares. In support of our findings, it has been reported that serum miR-433-3p expression is upregulated in Day 19 and 24 pregnant cows [34] and during the first trimester of pregnancy in women [43]. Moreover, it has been reported that serum $h s a-m i R-1247-3 p$ expression is reduced in women with ectopic pregnancy [37] and preeclampsia [39] in comparison to normal pregnant women. To further assess the accuracy of eca-miR-1247-3p, eca-miR-134-5p, eca-miR-382-5p, and eca-miR-433-3p in differentiating pregnant (25 d) from non-pregnant mares, ROC analysis was performed and indicated that eca-miR-1247-3p followed by eca-miR-134-5p retain the highest accuracy. It is worth noting that miR-134-5p is highly expressed during embryonic development and becomes restricted to the brain postnatally [44,45]. Moreover, it has been reported that $m i R-134-5 p$ is uniquely correlated in maternal and fetal circulation in women [46]. These facts might suggest that the serum enrichment with eca-miR-134-5p during Days 25 and 45 of equine pregnancy is of embryonic origin. Altogether, our findings suggest that eca-miR-1247-3p and eca-miR-134-5p could be potential biomarkers for pregnancy establishment and/or early embryonic loss at 25 days of equine pregnancy. In equine, it has been suggested that changes in circulating miRNAs could be detected as early as Days 9-13 of pregnancy [47]. However, eca-miR-1247-3p and eca-miR-134-5p profiles were not investigated in the previously mentioned study. Therefore, further studies are needed to elucidate the expression profile of eca-miR-1247-3p and eca-miR-134-5p during earlier stages of equine pregnancy.

In the current study, circulating eca-miR-1247-3p, eca-miR-134-5p, eca-miR-409-3p, and eca-miR-379-5p expression profile were enriched in $45 \mathrm{~d}$ pregnant mares in comparison to non-pregnant. Moreover, ROC analysis indicated that eca-miR-1247-3p and eca-miR-409-3p have retained the highest accuracy in distinguishing $45 \mathrm{~d}$ pregnant mares from non-pregnant mares. Again, these findings suggest that $e c a-m i R-1247-3 p$ and $e c a-m i R-409-3 p$ could be potential biomarkers for pregnancy establishment and/or early pregnancy loss at Day 45 of equine pregnancy.

The serum enrichment with eca-miR-1247-3p, eca-miR-134-5p, eca-miR-382-5p, and eca-miR-433-3p at $25 \mathrm{~d}$ pregnancy as well as eca-miR-1247-3p, eca-miR-134-5p, eca-miR-409-3p, and eca-miR-379-5p at 
$45 \mathrm{~d}$ pregnancy suggest that these miRNAs are involved in early pregnancy events. In the same line, we found a similar pattern in the expression profile of this cluster of miRNAs in equine chorioallantoic membranes [29]. Moreover, a high expression of C24MC at $45 \mathrm{~d}$ was followed by a gradual decline toward the end of the gestation. To gain further insight into the biology of these miRNAs' targets, GO enrichment (biological process) analysis and pathway analysis were carried out. GO analysis revealed that these miRNAs are mainly implicated in nervous system development as well as organ development, cell-cell signaling, and Wnt signaling pathway, among others. In support of our outcomes, it has been reported that the expression of both $m i R-1247-3 p$ and $m i R-409-3 p$ is significantly downregulated in embryonic neuroblastoma [48,49]. In line with this, ISH analysis of eca-miR-409-3p revealed extensive expression in various tissues of the developing embryo including the central nervous system, among others. Moreover, pathway analysis of the same set of miRNAs revealed that they are associated with Wnt signaling pathway and oxytocin receptor mediated signaling pathway, among others. Interestingly, it has been reported that oxytocin responsiveness is altered during early equine pregnancy, and reduced expression of the oxytocin receptor (OXTR) seems to be regulated at the posttranscriptional level rather than the transcriptional level [50]. Our prediction suggests that these miRNAs are potentially involved in oxytocin receptor mediated signaling pathway during early pregnancy in the mare.

In conclusion, the present study is the first to elucidate the maternal circulation profile of C24MC-associated miRNAs throughout equine pregnancy in comparison to non-pregnant (diestrus) and postpartum mares. Moreover, this study introduced serum eca-miR-1247-3p, eca-miR-134-5p, and eca-miR409-3p as possible non-invasive biomarkers for early pregnancy ( 25 and $45 \mathrm{~d}$ ) in mare. We further localized one of these miRNAs (eca-miR-409-3p) in the equine $25 \mathrm{~d}$ embryo, demonstrating the expression of it in the central nervous system and developing organs. Overall, the high abundance of these embryo-derived miRNAs in the maternal circulation suggests an embryo-maternal communication during the equine early pregnancy.

\section{Material and Methods}

\subsection{Animal Use}

All animal procedures were prospectively approved by and completed in accordance with the Institutional Animal Care and Use Committee of the University of Kentucky (Approval No. \#2014-1341, Date: 15 January 2015). All horses used in this study were mixed-breed, ranging between 250 and 550 $\mathrm{kg}$. Mares were housed on pasture with ad libitum grass hay. Gestational age (GA) was determined based on the day of ovulation (Day 0).

\subsection{Serum Collection and Preparation}

For this cross-sectional study, blood sample from pregnant mares at 25 days ( $25 \mathrm{~d} ; n=5), 45 \mathrm{~d}$ $(n=9), 4$ months $(4 \mathrm{mo} ; n=7), 6 \mathrm{mo}(n=9)$, and $10 \mathrm{mo}(n=6)$ of gestation was collected via jugular venipuncture. Similarly, blood samples were collected from diestrus (Day 7-9 post ovulation, $n=14$ ) and postpartum (immediately after normal parturition, $n=3$ ). After clotting, the samples were centrifuged at $500 \times g$ for $10 \mathrm{~min}$ at $4{ }^{\circ} \mathrm{C}$. Serum was subsequently removed and stored at $-20^{\circ} \mathrm{C}$.

\subsection{MiRNA Extraction, $c D N A$ Synthesis, and RT-qPCR}

MiRNAs were extracted from serum samples $(400 \mu \mathrm{L})$ using the miRNeasy Micro Kit (Qiagen, Hilden, Germany) according to the manufacturer's instructions with the following modification: TRIzol $^{\mathrm{TM}}$ LS (Life technologies, Carlsbad, CA, USA) was used as the lysis reagent; $200 \mu \mathrm{L}$ of chloroform was used instead of $140 \mu \mathrm{L}$; and the final elution step was performed using $20 \mu \mathrm{L}$ of RNase-free water instead of $14 \mu \mathrm{L}$. Complementary DNA synthesis was carried out using the miScript II RT Kit (Qiagen). Three $\mu \mathrm{L}$ of cDNA product from each sample were combined to make the pooled serum samples. Pooled samples were only used as RT-qPCR inter-plate controls. The expression 
of mature miRNAs was determined by RT-qPCR using the miScript SYBR Green PCR kits (Qiagen, Hilden, Germany) with the miScript Universal Primer along with miRNA-specific primers according to the manufacturer's guidelines. Primers were designed using miRprimer software (version 2.0) for the candidate miRNAs [51].

The expression of the C24MC-associated miRNAs $(n=26)$, which were previously tested in the equine CAM throughout gestation [29], was evaluated in the collected serum samples. The list of miRNA candidates and primer sequences is provided in Table 2 . The primers' efficiency was verified on the pooled samples. Primers with CT values $<35$ that did not produce primer-dimers were used for further experimentation. Otherwise, primers were re-designed and re-tested. The primers that did not yield amplified PCR products in all the samples from at least one of the time-points were excluded from further analysis. Eca-miR-10a (gcagtaccetgtagatccga), eca-miR-21 (gcagtagcttatcagactgatg), and eca-Let-7a (gcagtgaggtagtaggttg) were used as reference genes for serum [52]. A DNA melting curve was generated to discriminate between specific and non-specific amplification products. Real-Time qPCR was performed in triplicate for all samples [53,54]. PCR efficiencies were calculated using LinRegPCR (version 2012.0; http://www.hartfaalcentrum.nl) to ensure that all primers resulted in PCR efficiencies between 1.8 and 2.1.

Table 2. List of selected C24MC-associated miRNAs evaluated by RT-qPCR and their respective primer sequences.

\begin{tabular}{|c|c|c|c|}
\hline MiRNA ID & Accession ID * & Mature Sequence & Forward Primer \\
\hline$e c a-m i R-127-5 p$ & MIMAT0004604 & cugaagcucagagggcucugau & ctgaagctcagagggct \\
\hline eca-miR-134-5p & MIMAT0013127 & ugugacugguugaccagagggg & gcagtgtgactggttgac \\
\hline eca-miR-323-3p & MIMAT0013132 & cacauuacacggucgaccucu & gcagcacattacacggt \\
\hline eca-miR-323-5p & MIMAT0013131 & aggugguccguggcgcguucgc & ccgtggcgcgtt \\
\hline eca-miR-369-3p & MIMAT0013141 & aauaauacaugguugaucuuu & cagcgcagaataatacatggt \\
\hline eca-miR-370-3p & MIMAT0013142 & gccugcugggguggaaccuggu & cctgctggggtgga \\
\hline eca-miR-370-5p & MIMAT0026483 & caggucacgucucugcaguuac & cagcaggtcacgtctct \\
\hline eca-miR-379-5p & MIMAT0013147 & ugguagacuauggaacguagg & cagtggtagactatggaacg \\
\hline eca-miR-382-5p & MIMAT0013150 & gaaguuguucgugguggauucg & aggaagttgttcgtggtg \\
\hline eca-miR-3958-3p & MIMAT0034486 & cagauauugcacgguugaucucuu & gcagatattgcacggttga \\
\hline eca-miR-3958-5p & MIMAT0019275 & agguuguccgugauguauuugc & agaggttgtccgtgatgt \\
\hline eca-miR-409-3p & MIMAT0013152 & gaauguugcucggugaaccсcu & aggaatgttgctcggtga \\
\hline eca-miR-411-3p & MIMAT0013154 & uauguaacacgguccacuaacc & cagtatgtaacacggtccac \\
\hline eca-miR-411-5p & MIMAT0003329 & uaguagaccguauagcguacg & cagtagtagaccgtatagcgt \\
\hline eca-miR-412-3p & MIMAT0013155 & uucaccugguccacuagccg & gcagttcacctggtcca \\
\hline eca-miR-412-5p & MIMAT0026557 & uggucgaccaguuggaaaguaau & cagtggtcgaccagttg \\
\hline eca-miR-432-5p & MIMAT0013157 & ucuuggaguaggucauugggugg & cagtcttggagtaggtcattg \\
\hline eca-miR-433-3p & MIMAT0013158 & aucaugaugggcuccucggugu & catgatgggctcctcg \\
\hline eca-miR-485-3p & MIMAT0013160 & gucauacacggcucuccucucu & gcaggtcatacacggct \\
\hline eca-miR-485-5p & MIMAT0013159 & agaggcuggccgugaugaauuc & ggctggccgtgatga \\
\hline$e c a-m i R-487 a-5 p$ & MIMAT0026559 & gugguuaucccugcuguguucg & caggtggttatccctgct \\
\hline$e c a-m i R-487 b-3 p$ & MIMAT0013162 & aaucguacagggucauccacuu & cagaatcgtacagggtcatc \\
\hline$e c a-m i R-493 b-5 p$ & MIMAT0002813 & uuguacaugguaggcuuucauu & gcgcagttgtacatggtag \\
\hline eca-miR-543-3p & MIMAT0013169 & aaacauucgcggugcacuucuu & gcagaaacattcgcggtg \\
\hline$h s a-m i R-1247-3 p$ & MIMAT0022721 & ccccgggaacgucgagacuggagc & cgggaacgtcgagac \\
\hline$h s a-m i R-154-5 p$ & MIMAT0000452 & uagguuauccguguugccuucg & gcagtaggttatccgtgttg \\
\hline
\end{tabular}




\subsection{Target Prediction for the miRNA Which Were Highly Expressed during Early Pregnancy ( 25 and $45 d$ )}

Predicted targets for the six highly expressed miRNAs at 25 and $45 \mathrm{~d}$ were selected from IPA (only the Experimentally Observed or High Predicted) and miRDB.org (Target Score $>80$ ). Next, to predict functions of C24MC-associated miRNAs, the biological functions and physiological pathways of the target mRNA were analyzed by DAVID and Protein ANalysis THrough Evolutionary Relationships Classification System (PANTHER; Release 13.1) ontology classification system, respectively [55,56].

\subsection{Eca-miR-409-3p Localization by In Situ Hybridization}

The embryo ( $25 \mathrm{~d}$ ) and extra-embryonic membranes ( 25 and $45 \mathrm{~d}$ ) were retrieved by uterine lavage [29]. The tissue samples were fixed in $10 \%$ formaldehyde for $24 \mathrm{~h}$, transferred to $70 \%$ methanol and paraffin embedded [29]. The expression of eca-miR-409-3p was investigated by chromogenic ISH using a dual digoxigenin (DIG)-labeled LNA ${ }^{\mathrm{TM}}$ probe specific to hsa-miR-409-3p (610701-360; miRCURY LNA ${ }^{\mathrm{TM}}$, Exiqon, Vedbaek, Denmark) as previously described [29] on 25 d embryos ( $\mathrm{n}=3$ ) along with the extra-embryonic membranes. A dual DIG-labeled LNA ${ }^{\mathrm{TM}}$ probe specific to U6 snRNA (\#699002-360; Exiqon) and a scrambled miRNA probe (\#699003-360; Exiqon) were used as positive and negative controls, respectively.

\subsection{Data Analysis}

Delta $\mathrm{CT}(\Delta \mathrm{CT})$ values for serum samples were calculated where $\Delta \mathrm{CT}=$ (the $\mathrm{CT}$ values of the miRNA of interest-the CT values of all three reference miRNAs [geometric mean]) [57,58]. Results are presented as $-\Delta \mathrm{CT}$ (negative $\Delta \mathrm{CT}$ is more intuitive than $\Delta \mathrm{CT}$ ). The expressions of $\mathrm{C} 24 \mathrm{MC}$-associated miRNAs were compared across GA, postpartum and with samples from non-pregnant mares using one-way analysis of variance (one-way ANOVA) followed by a pairwise comparison of means using T-test. Significance was set at $p<0.05$. Pearson's correlation was performed to analyze the relationship between the expression profile of all the tested miRNAs. Receiver operating characteristic (ROC) curve analysis was carried out to assess the accuracy of eca-miR-1247-3p, eca-miR-134-5p, eca-miR-382-5p, and eca-miR-433-3p (differentially expressed miRNAs at $25 \mathrm{~d}$ ) in differentiating pregnant ( $25 \mathrm{~d}$ ) from non-pregnant (diestrus) mares. Moreover, ROC curve analysis was carried out to assess the accuracy of eca-miR-1247-3p, eca-miR-134-5p, eca-miR-409-3p, and eca-miR-379-5p (differentially expressed miRNAs at $45 \mathrm{~d}$ ) in distinguishing pregnant (45 d) from non-pregnant (diestrus) mares. The area under the ROC curve $\left(A U C_{R O C}\right)$ was estimated and used to evaluate the accuracy of the tested miRNAs $[59,60]$. In brief, the $A U C_{R O C}$ was inferred as noninformative $\left(A U C_{R O C} \leq 0.5\right)$, poorly accurate $\left(0.5<A_{R U C}\right.$ $\leq 0.7)$, moderately accurate $\left(0.7<\mathrm{AUC}_{\mathrm{ROC}} \leq 0.9\right)$, highly accurate $\left(0.9<\mathrm{AUC}_{\mathrm{ROC}}<1\right)$, or perfect $\left(A U C_{R O C}=1\right)[61]$. The $A U C_{R O C}$ for each parameter was compared with the expected value $\left(A U C_{R O C}\right.$ $=0.5$ ) under the null hypothesis of a noninformative test.

Author Contributions: P.D. (Pouya Dini) designed the study, performed the experimental procedures, statistical analyses, visualization, and contributed to manuscript preparation. H.E.-S.A. performed statistical analyses, visualization, conceptualization, and manuscript writing. M.C. performed ISH analysis, visualization, and revised the manuscript. S.C.L. contributed to study design, conceptualization, and revision of the manuscript. A.E.-V. provided assistance for the experimental procedure and contributed to the revision of the manuscript. K.E.S. provided assistance for the experimental procedure and contributed to the revision of the manuscript. P.D. (Peter Daels) oversaw the entire project, including acquisition of funding (BOF), experimental design, and review of the manuscript. B.A.B. oversaw the entire project, including the acquisition of funding, revision of the manuscript, and submission. All authors read and approved the final manuscript.

Funding: This work was funded by the Special Research Fund (BOF) at Ghent University, the Albert G. Clay Endowment, and the Paul Mellon Postdoctoral fellowships at the University of Kentucky.

Conflicts of Interest: The authors declare that they have no competing interests.

\section{References}

1. Bartel, D.P. MicroRNAs: Genomics, biogenesis, mechanism, and function. Cell 2004, 116, 281-297. [CrossRef] 
2. Krol, J.; Sobczak, K.; Wilczynska, U.; Drath, M.; Jasinska, A.; Kaczynska, D.; Krzyzosiak, W.J. Structural features of microRNA (miRNA) precursors and their relevance to miRNA biogenesis and small interfering RNA/short hairpin RNA design. J. Biol. Chem. 2004, 279, 42230-42239. [CrossRef] [PubMed]

3. Cheng, A.M.; Byrom, M.W.; Shelton, J.; Ford, L.P. Antisense inhibition of human miRNAs and indications for an involvement of miRNA in cell growth and apoptosis. Nucleic Acids Res. 2005, 33, 1290-1297. [CrossRef] [PubMed]

4. Bueno, M.J.; Perez de Castro, I.; Malumbres, M. Control of cell proliferation pathways by microRNAs. Cell Cycle 2008, 7, 3143-3148. [CrossRef]

5. Ardekani, A.M.; Naeini, M.M. The Role of MicroRNAs in Human Diseases. Avicenna J. Med. Biotechnol. 2010, 2, 161-179.

6. Ventura, W.; Koide, K.; Hori, K.; Yotsumoto, J.; Sekizawa, A.; Saito, H.; Okai, T. Placental expression of microRNA-17 and-19b is down-regulated in early pregnancy loss. Eur. J. Obstet. Gynecol. Reprod. Biol. 2013, 169, 28-32. [CrossRef]

7. Zhao, Z.; Moley, K.H.; Gronowski, A.M. Diagnostic potential for miRNAs as biomarkers for pregnancy-specific diseases. Clin. Biochem. 2013, 46, 953-960. [CrossRef]

8. Kotlabova, K.; Doucha, J.; Hromadnikova, I. Placental-specific microRNA in maternal circulation-Identification of appropriate pregnancy-associated microRNAs with diagnostic potential. J. Reprod. Immunol. 2011, 89, 185-191. [CrossRef]

9. Lai, X.; Vera, J. MicroRNA Clusters. In Encyclopedia of Systems Biology; Dubitzky, W., Wolkenhauer, O., Cho, K.-H., Yokota, H., Eds.; Springer: New York, NY, USA, 2013; pp. 1310-1314.

10. Weber, M.J. New human and mouse microRNA genes found by homology search. FEBS J. 2005, 272, 59-73. [CrossRef]

11. Liang, Y.; Ridzon, D.; Wong, L.; Chen, C. Characterization of microRNA expression profiles in normal human tissues. BMC Genom. 2007, 8, 166. [CrossRef]

12. Ghai, V.; Wang, K. Recent progress toward the use of circulating microRNAs as clinical biomarkers. Arch. Toxicol. 2016, 90, 2959-2978. [CrossRef] [PubMed]

13. Pritchard, C.C.; Cheng, H.H.; Tewari, M. MicroRNA profiling: Approaches and considerations. Nat. Rev. Genet. 2012, 13, 358-369. [CrossRef] [PubMed]

14. Gantier, M.P.; McCoy, C.E.; Rusinova, I.; Saulep, D.; Wang, D.; Xu, D.; Irving, A.T.; Behlke, M.A.; Hertzog, P.J.; Mackay, F.; et al. Analysis of microRNA turnover in mammalian cells following Dicer1 ablation. Nucleic Acids Res. 2011, 39, 5692-5703. [CrossRef] [PubMed]

15. Xourgia, E.; Papazafiropoulou, A.; Melidonis, A. Circulating microRNAs as biomarkers for diabetic neuropathy: A novel approach. World J. Exp. Med. 2018, 8, 18-23. [CrossRef] [PubMed]

16. Singh, R.; Ramasubramanian, B.; Kanji, S.; Chakraborty, A.R.; Haque, S.J.; Chakravarti, A. Circulating microRNAs in cancer: Hope or hype? Cancer Lett. 2016, 381, 113-121. [CrossRef] [PubMed]

17. He, Y.; Lin, J.; Kong, D.; Huang, M.; Xu, C.; Kim, T.K.; Etheridge, A.; Luo, Y.; Ding, Y.; Wang, K. Current State of Circulating MicroRNAs as Cancer Biomarkers. Clin. Chem. 2015, 61, 1138-1155. [CrossRef]

18. Zhou, S.-S.; Jin, J.-P.; Wang, J.-Q.; Zhang, Z.-G.; Freedman, J.H.; Zheng, Y.; Cai, L. miRNAS in cardiovascular diseases: Potential biomarkers, therapeutic targets and challenges. Acta Pharmacol. Sin. 2018, 39, 1073-1084. [CrossRef]

19. Kaneto, C.M.; Nascimento, J.S.; Prado, M.; Mendonca, L.S.O. Circulating miRNAs as biomarkers in cardiovascular diseases. Eur. Rev. Med. Pharm. Sci. 2019, 23, 2234-2243.

20. Hackl, M.; Heilmeier, U.; Weilner, S.; Grillari, J. Circulating microRNAs as novel biomarkers for bone diseases-Complex signatures for multifactorial diseases? Mol. Cell. Endocrinol. 2016, 432, 83-95. [CrossRef]

21. Morales-Prieto, D.M.; Ospina-Prieto, S.; Chaiwangyen, W.; Schoenleben, M.; Markert, U.R. Pregnancy-associated miRNA-clusters. J. Reprod. Immunol. 2013, 97, 51-61. [CrossRef]

22. Ioannidis, J.; Donadeu, F.X. Circulating microRNA Profiles during the Bovine Oestrous Cycle. PLoS ONE 2016, 11, e0158160. [CrossRef] [PubMed]

23. Da Silveira, J.C.; Veeramachaneni, D.N.; Winger, Q.A.; Carnevale, E.M.; Bouma, G.J. Cell-secreted vesicles in equine ovarian follicular fluid contain miRNAs and proteins: A possible new form of cell communication within the ovarian follicle. Biol. Reprod. 2012, 86, 71. [CrossRef] [PubMed]

24. Sohel, M.M.; Hoelker, M.; Noferesti, S.S.; Salilew-Wondim, D.; Tholen, E.; Looft, C.; Rings, F.; Uddin, M.J.; Spencer, T.E.; Schellander, K.; et al. Exosomal and Non-Exosomal Transport of Extra-Cellular microRNAs in 
Follicular Fluid: Implications for Bovine Oocyte Developmental Competence. PLoS ONE 2013, 8, e78505. [CrossRef] [PubMed]

25. Liang, J.; Wang, S.; Wang, Z. Role of microRNAs in embryo implantation. Reprod. Biol. Endocrinol. 2017, 15, 90. [CrossRef]

26. Reza, A.M.M.T.; Choi, Y.-J.; Han, S.G.; Song, H.; Park, C.; Hong, K.; Kim, J.-H. Roles of microRNAs in mammalian reproduction: From the commitment of germ cells to peri-implantation embryos. Biol. Rev. 2019, 94, 415-438. [CrossRef]

27. Nothnick, W.B. The role of micro-RNAs in the female reproductive tract. Reproduction 2012, 143, 559-576. [CrossRef]

28. Hayder, H.; O’Brien, J.; Nadeem, U.; Peng, C. MicroRNAs: Crucial regulators of placental development. Reproduction 2018, 155, R259-R271. [CrossRef]

29. Dini, P.; Daels, P.; Loux, S.C.; Esteller-Vico, A.; Carossino, M.; Scoggin, K.E.; Ball, B.A. Kinetics of the chromosome 14 microRNA cluster ortholog and its potential role during placental development in the pregnant mare. BMC Genom. 2018, 19, 954. [CrossRef]

30. Prieto, D.M.M.; Markert, U.R. MicroRNAs in pregnancy. J. Reprod. Immunol. 2011, 88, 106-111. [CrossRef]

31. Tsochandaridis, M.; Nasca, L.; Toga, C.; Levy-Mozziconacci, A. Circulating microRNAs as clinical biomarkers in the predictions of pregnancy complications. BioMed Res. Int. 2015, 2015. [CrossRef]

32. Cretoiu, D.; Xu, J.; Xiao, J.; Suciu, N.; Cretoiu, S.M. Circulating MicroRNAs as Potential Molecular Biomarkers in Pathophysiological Evolution of Pregnancy. Dis. Markers 2016, 2016, 3851054. [CrossRef] [PubMed]

33. Ouyang, Y.; Mouillet, J.F.; Coyne, C.B.; Sadovsky, Y. Review: Placenta-specific microRNAs in exosomes-Good things come in nano-packages. Placenta 2014, 35, S69-S73. [CrossRef] [PubMed]

34. Gebremedhn, S.; Salilew-Wondim, D.; Hoelker, M.; Held-Hoelker, E.; Neuhoff, C.; Tholen, E.; Schellander, K.; Tesfaye, D. Exploring maternal serum microRNAs during early pregnancy in cattle. Theriogenology 2018, 121, 196-203. [CrossRef] [PubMed]

35. Yang, Q.; Gu, W.W.; Gu, Y.; Yan, N.N.; Mao, Y.Y.; Zhen, X.X.; Wang, J.M.; Yang, J.; Shi, H.J.; Zhang, X.; et al. Association of the peripheral blood levels of circulating microRNAs with both recurrent miscarriage and the outcomes of embryo transfer in an in vitro fertilization process. J. Transl. Med. 2018, 16, 186. [CrossRef] [PubMed]

36. Hosseini, M.K.; Gunel, T.; Gumusoglu, E.; Benian, A.; Aydinli, K. MicroRNA expression profiling in placenta and maternal plasma in early pregnancy loss. Mol. Med. Rep. 2018, 17, 4941-4952. [CrossRef] [PubMed]

37. Zhang, S.; Sun, Q.; Jiang, X.; Gao, F. Clinical significance of expression of hsa-mir-1247 and hsa-mir-1269a in ectopic pregnancy due to salpingitis. Exp. Ther. Med. 2018, 15, 4901-4905. [CrossRef] [PubMed]

38. Kontomanolis, E.N.; Kalagasidou, S.; Fasoulakis, Z. MicroRNAs as Potential Serum Biomarkers for Early Detection of Ectopic Pregnancy. Cureus 2018, 10, e2344. [CrossRef]

39. Enquobahrie, D.A.; Abetew, D.F.; Sorensen, T.K.; Willoughby, D.; Chidambaram, K.; Williams, M.A. Placental microRNA expression in pregnancies complicated by preeclampsia. Am. J. Obstet. Gynecol. 2011, 204, 178.e12-178.e21. [CrossRef]

40. Awamleh, Z.; Gloor, G.B.; Han, V.K.M. Placental microRNAs in pregnancies with early onset intrauterine growth restriction and preeclampsia: Potential impact on gene expression and pathophysiology. BMC Med Genom. 2019, 12, 91. [CrossRef]

41. Loux, S.C.; Fernandes, C.B.; Dini, P.; Wang, K.; Wu, X.; Baxter, D.; Scoggin, K.E.; Troedsson, M.H.T.; Squires, E.L.; Ball, B.A. Small RNA (sRNA) expression in the chorioallantois, endometrium and serum of mares following experimental induction of placentitis. Reprod. Fertil. Dev. 2019, 31, 1141-1156. [CrossRef]

42. Wommack, J.C.; Trzeciakowski, J.P.; Miranda, R.C.; Stowe, R.P.; Ruiz, R.J. Micro RNA clusters in maternal plasma are associated with preterm birth and infant outcomes. PLoS ONE 2018, 13, e0199029. [CrossRef] [PubMed]

43. Miura, K.; Miura, S.; Yamasaki, K.; Higashijima, A.; Kinoshita, A.; Yoshiura, K.-I.; Masuzaki, H. Identification of Pregnancy-Associated MicroRNAs in Maternal Plasma. Clin. Chem. 2010, 56, 1767-1771. [CrossRef] [PubMed]

44. Schratt, G.M.; Tuebing, F.; Nigh, E.A.; Kane, C.G.; Sabatini, M.E.; Kiebler, M.; Greenberg, M.E. A brain-specific microRNA regulates dendritic spine development. Nature 2006, 439, 283-289. [CrossRef] 
45. Landgraf, P.; Rusu, M.; Sheridan, R.; Sewer, A.; Iovino, N.; Aravin, A.; Pfeffer, S.; Rice, A.; Kamphorst, A.O.; Landthaler, M.; et al. A mammalian microRNA expression atlas based on small RNA library sequencing. Cell 2007, 129, 1401-1414. [CrossRef] [PubMed]

46. Williams, Z.; Ben-Dov, I.Z.; Elias, R.; Mihailovic, A.; Brown, M.; Rosenwaks, Z.; Tuschl, T. Comprehensive profiling of circulating microRNA via small RNA sequencing of cDNA libraries reveals biomarker potential and limitations. Proc. Natl. Acad. Sci. USA 2013, 110, 4255-4260. [CrossRef]

47. Klohonatz, K.M.; Cameron, A.D.; Hergenreder, J.R.; da Silveira, J.C.; Belk, A.D.; Veeramachaneni, D.N.; Bouma, G.J.; Bruemmer, J.E. Circulating miRNAs as Potential Alternative Cell Signaling Associated with Maternal Recognition of Pregnancy in the Mare. Biol. Reprod. 2016, 95, 124. [CrossRef]

48. Urdinguio, R.G.; Fernandez, A.F.; Lopez-Nieva, P.; Rossi, S.; Huertas, D.; Kulis, M.; Liu, C.G.; Croce, C.M.; Calin, G.A.; Esteller, M. Disrupted microRNA expression caused by Mecp2 loss in a mouse model of Rett syndrome. Epigenetics 2010, 5, 656-663. [CrossRef]

49. Wu, T.; Lin, Y.; Xie, Z. MicroRNA-1247 inhibits cell proliferation by directly targeting ZNF346 in childhood neuroblastoma. Biol. Res. 2018, 51, 13. [CrossRef]

50. Klein, C. Pregnancy Recognition and Implantation of the Conceptus in the Mare. Adv. Anat. Embryol. Cell Biol. 2015, 216, 165-188.

51. Busk, P.K. A tool for design of primers for microRNA-specific quantitative RT-qPCR. BMC Bioinform. 2014, 15, 29. [CrossRef]

52. Dini, P.; Loux, S.C.; Scoggin, K.E.; Esteller-Vico, A.; Squires, E.L.; Troedsson, M.H.T.; Daels, P.; Ball, B.A. Identification of Reference Genes for Analysis of microRNA Expression Patterns in Equine Chorioallantoic Membrane and Serum. Mol. Biotechnol. 2018, 60, 62-73. [CrossRef] [PubMed]

53. Bellingham, S.A.; Shambrook, M.; Hill, A.F. Quantitative Analysis of Exosomal miRNA via qPCR and Digital PCR. Methods Mol. Biol. 2017, 1545, 55-70. [PubMed]

54. Salone, V.; Rederstorff, M. Stem-loop RT-PCR based quantification of small non-coding RNAs. Methods Mol. Biol. 2015, 1296, 103-108. [PubMed]

55. Thomas, P.D.; Campbell, M.J.; Kejariwal, A.; Mi, H.; Karlak, B.; Daverman, R.; Diemer, K.; Muruganujan, A.; Narechania, A. PANTHER: A library of protein families and subfamilies indexed by function. Genome Res. 2003, 13, 2129-2141. [CrossRef] [PubMed]

56. Dennis, G.; Sherman, B.T.; Hosack, D.A.; Yang, J.; Gao, W.; Lane, H.C.; Lempicki, R.A. DAVID: Database for annotation, visualization, and integrated discovery. Genome Biol. 2003, 4, R60. [CrossRef]

57. Wang, Z.; Yang, B. MicroRNA expression Detection Methods; Springer: Berlin/Heidelberg, Germany, 2009.

58. Vandesompele, J.; De Preter, K.; Pattyn, F.; Poppe, B.; Van Roy, N.; De Paepe, A.; Speleman, F. Accurate normalization of real-time quantitative RT-PCR data by geometric averaging of multiple internal control genes. Genome Biol. 2002, 3, Research0034. [CrossRef]

59. Greiner, M.; Pfeiffer, D.; Smith, R. Principles and practical application of the receiver-operating characteristic analysis for diagnostic tests. Prev. Vet. Med. 2000, 45, 23-41. [CrossRef]

60. Hanley, J.A.; McNeil, B.J. The meaning and use of the area under a receiver operating characteristic (ROC) curve. Radiology 1982, 143, 29-36. [CrossRef]

61. Swets, J.A. Measuring the accuracy of diagnostic systems. Science 1988, 240, 1285-1293. [CrossRef]

(C) 2019 by the authors. Licensee MDPI, Basel, Switzerland. This article is an open access article distributed under the terms and conditions of the Creative Commons Attribution (CC BY) license (http://creativecommons.org/licenses/by/4.0/). 